\title{
Parasexual Cycle Provides Genetic Segregants Equivalent to Sexual Progeny in the Rice Blast Fungus Magnaporthe oryzae
}

\author{
Masako Tsujimoto NOGUCHI ${ }^{1 *}$, Nobuko YASUDA ${ }^{2}$ and Yoshikatsu FUJITA ${ }^{2}$ \\ ${ }^{1}$ National Agricultural Research Center, Hokuriku Area (Joetsu, Niigata 943-0193, Japan) \\ ${ }^{2}$ National Agricultural Research Center (Tsukuba, Ibaraki 305-8666, Japan)
}

\begin{abstract}
We demonstrated that the segregation ratio of avirulence and virulence traits on rice cultivars, Hattan 3 and line K59-1, and the mating types of parasexual recombinants were consistent with those of sexual progeny in the rice blast fungus Magnaporthe oryzae. Our results indicated that the genetic characters of parasexual recombinants segregated in a recombination manner similar to sexual events.
\end{abstract}

Discipline: Plant disease

Additional key word: Pyricularia oryzae

\section{Introduction}

Blast caused by Magnaporthe oryzae B. Couch (anamorph Pyricularia oryzae Cavara), previously known as Magnaporthe grisea (Hebert) Barr ${ }^{4}$, is the most serious disease of rice and limits rice production worldwide. Resistant cultivars are usually effective for controlling the disease, although newly developed resistant cultivars have been affected within several years after their introduction. The breakdown of their blast resistance results from the occurrence of pathogenic variants (races). Sexual mating, mutation, and parasexual recombination are thought to be factors affecting diversification and variability of the pathogen. Since ascospore production from a cross between Japanese field isolates had never been reported, mutation and parasexual recombination are likely the two major causes of variation in the pathogenicity of $M$. oryzae.

The rice cultivar specificity in this fungus has a genefor-gene relationship ${ }^{7}$, and avirulence genes have been reported $^{11,12,19,23,24}$. While numerous studies on the parasexual cycle have been conducted in the rice blast fungus $^{3,5,6,8,13,14,22,25}$, no genetic analyses have been performed on parasexual recombinants that included avirulence genes. Therefore, we carried out a genetic study after obtaining parasexual recombinants from co-cultures of two different antibiotic-resistant isolates of the fungus: isolate Y90-71BI derived from introducing plasmid pBARKS1, a bialaphos (BI)-resistance vector that contains the Ignite/Basta-resistance ( $b a r)$ gene ${ }^{1,16}$, into isolate Y90-71, and isolate 3514-R-2BS derived from introducing plasmid pBF101, a blasticidin S (BS)-resistance vector that contains the blasticidin $\mathrm{S}$ deaminase gene $(B S D)^{9}$, into isolate $3514-\mathrm{R}-2^{15}$. We revealed that the segregation of avirulence to virulence ( $a v r / v i r)$ in the progeny derived from crossing Y90-71 and 3514-R-2 on Hattan $3^{24}$ and line K59-1 (unpublished data) was in a 1:1 ratio. The segregation of avr/vir in the parasexual recombinants (BI-BSresistant parasexual recombinants) was consistent with a $1: 1$ ratio on line K59-1, but not on Hattan $3^{15}$. We postulated that the segregation of avr/vir of the BI-BS-resistant parasexual recombinants on Hattan 3 was consistent with that of sexual progeny and that the antibiotic-resistance genes and genes for virulence on Hattan 3 might be linked as a result of transformation. To eliminate the confounding effect of linkage, in this study, we compared the segregation of avr/vir in the BI-BS-resistant parasexual recombinants with that in the BI-BS-resistant sexual progeny derived from crossing Y90-71BI and 3514-R-2BS on Hattan 3 and line K59-1. In addition, we examined the segregation of the mating types in the BI-BS-resistant sexual progeny derived from crossing Y90-71BI and 3514-R-2BS.

\footnotetext{
Present address:

${ }^{1}$ National Institute for Agro-Environmental Sciences, Tsukuba, Ibaraki 305-8604, Japan

*Corresponding author: e-mail macha@affrc.go.jp

Received 7 September 2006; accepted 19 October 2006.
} 


\section{Materials and methods}

\section{Magnaporthe oryzae isolates and plant materials}

Magnaporthe oryzae isolates and plant materials used in this study are listed in Table 1.

\section{Crosses of blast isolates}

Y90-71BI and 3514-R-2BS were crossed following the procedure described by Yaegashi ${ }^{21}$ with modifications. Perithecia resulting from crossing were crushed with a scalpel to release ascospores, which were transferred to potato dextrose agar (PDA) containing BI $(800 \mu \mathrm{g} / \mathrm{ml})$ and BS $(100 \mu \mathrm{g} / \mathrm{ml})$ with a pipette to obtain BI-BS-resistant sexual progeny. After incubation for 24 to $36 \mathrm{~h}$, singly germinated ascospores were separated with a micromanipulator and transferred to PDA.

\section{Mating type determination}

Mating type was determined by crossing $M$. oryzae isolates with tester isolates, Y93-164a-1 (MAT1-1, race 132) and Y93-245c-2 (MAT1-2, race 137), as described previously $^{15}$.

\section{Pathogenicity test}

The pathogenicity of the BI-BS-resistant sexual progeny was determined using a previously described method $^{15}$.

\section{Results}

\section{BI-BS-resistant sexual progeny}

Seventy sexual progeny resistant to BI and BS were obtained. All grew on PDA containing both antibiotics.

\section{Segregation of avirulence/virulence in BI-BS- resistant sexual progeny on Hattan 3 and line K59-1}

Y90-71 has Avr-Hattan 3 and was avirulent on Hattan 3, but virulent on line K59-1 carrying Pit. In contrast, 3514-R-2 has Avr-Pit and was virulent on Hattan 3, but avirulent on line K59-1. Inoculating Hattan 3 with the 70 BI-BS-resistant sexual progeny derived from the cross between Y90-71BI and 3514-R-2BS yielded 21 avirulent and 49 virulent isolates (Table 2). Inoculating line K59-1 with the 70 BI-BS-resistant sexual progeny yielded 43 avirulent and 27 virulent isolates. Inoculating Hattan 3

Table 1. Magnaporthe oryzae isolates and plant materials used in this study

\begin{tabular}{|c|c|c|}
\hline Isolates, plant materials & Relevant properties & References \\
\hline \multicolumn{3}{|l|}{ Magnaporthe oryzae isolates } \\
\hline Y90-71BI & $\mathrm{BI}^{\mathrm{r}}$, Avr-Hattan 3, MAT1-1, race 102 & Noguchi et al. ${ }^{15}$ \\
\hline 3514-R-2BS & $\mathrm{BS}^{\mathrm{r}}$, Avr-Pit, MAT1-2, race 136 & Noguchi et al. ${ }^{15}$ \\
\hline Y93-164a-1 & MAT1-1, race 132 & Noguchi et al. ${ }^{15}$ \\
\hline Y93-245c-2 & MAT1-2, race 137 & Noguchi et al. ${ }^{15}$ \\
\hline \multirow[t]{2}{*}{ BI-BS-resistant parasexual recombinants } & $\begin{array}{l}\text { Parasexual recombinants derived from co-cultures of Y90-71BI and } \\
\text { 3514-R-2BS }\end{array}$ & Noguchi et al. ${ }^{15}$ \\
\hline & $\mathrm{BI}^{\mathrm{r}}, \mathrm{BS}^{\mathrm{r}}$ & \\
\hline \multicolumn{3}{|l|}{ Plant materials } \\
\hline \multicolumn{3}{|l|}{ Oryza sativa } \\
\hline Hattan 3 & Japonica-type cultivar, Pik-s & Yasuda et al. ${ }^{24}$ \\
\hline Line K59-1 & One of the $\mathrm{F}_{3}$ lines from the cross between $\mathrm{K} 59$ and Norin 3, Pit & Noguchi et al. ${ }^{15}$ \\
\hline
\end{tabular}

Table 2. Segregation of avirulence to virulence on Hattan 3 and line K59-1 in BI-BS-resistant parasexual recombinants and in BI-BS-resistant sexual progeny

\begin{tabular}{|c|c|c|c|c|c|c|}
\hline \multirow[t]{2}{*}{ Rice plants } & \multicolumn{2}{|c|}{ Sexual progeny } & \multicolumn{2}{|c|}{ Parasexual recombinants ${ }^{\mathrm{a}}$} & \multirow[t]{2}{*}{$\chi^{2}$ value $^{\text {b) }}$} & \multirow[t]{2}{*}{ P value ${ }^{c)}$} \\
\hline & A & $\mathrm{V}$ & A & $\mathrm{V}$ & & \\
\hline Hattan 3 & 21 & 49 & 12 & 37 & 0.47 & $0.70-0.50$ \\
\hline Line K59-1 & 43 & 27 & 24 & 25 & 1.82 & $0.20-0.10$ \\
\hline
\end{tabular}

Pathogenicity was assayed 6 to 7 days after spraying conidial suspension on Hattan 3 and K59-1 seedlings ${ }^{15,24}$ (A: avirulence, V: virulence). a): Noguchi et al. ${ }^{15}$. b), c): Expected ratio, the ratio of avirulent to virulent in the BI-BS-resistant parasexual recombinants on Hattan 3 and line K59-1 to that in the BI-BS-resistant sexual progeny. 
Table 3. Segregation of mating types of BI-BS-resistant parasexual recombinants and BI-BS-resistant sexual progeny

\begin{tabular}{|c|c|c|c|c|}
\hline & \multicolumn{2}{|c|}{ Mating type $^{a)}$} & \multirow[t]{2}{*}{$\chi^{2}$ value $^{\text {b) }}$} & \multirow[t]{2}{*}{ P value ${ }^{c)}$} \\
\hline & MAT1-1 & MAT1-2 & & \\
\hline Sexual progeny & 26 & 34 & 0.002 & $0.98-0.95$ \\
\hline Parasexual recombinants ${ }^{\mathrm{d})}$ & 21 & 28 & & \\
\hline
\end{tabular}

a): Mating types were determined by crossing with tester isolates. b), c): Expected ratio, the ratio of the mating type in the BI-BSresistant parasexual recombinants to that in the BI-BS-resistant sexual progeny. d): Noguchi et al. ${ }^{15}$.

with the 49 BI-BS-resistant parasexual recombinants yielded 12 avirulent and 37 virulent isolates ${ }^{15}$. The chisquare value of 0.47 and $P$ value of $0.70-0.50$ indicated that the segregation of avirulence and virulence in the isolates fitted that in the BI-BS-resistant sexual progeny. Inoculating line K59-1 with the 49 BI-BS-resistant parasexual recombinants yielded 24 avirulent and 25 virulent isolates $^{15}$. The chi-square value of 1.82 and $P$ value of $0.20-0.10$ indicated that the ratio of avirulent to virulent in the BI-BS-resistant parasexual recombinants on line K59-1 was comparable with that in the BI-BS-resistant sexual progeny. The segregation ratio of avirulence and virulence on Hattan 3 and line K59-1 in the BI-BS-resistant parasexual recombinants was similar to that in the BI-BS-resistant progeny.

\section{Segregation of mating type of BI-BS-resistant sexual progeny of $M$. oryzae}

Sixty of the 70 BI-BS-resistant sexual progeny were used for mating type determination. Of the BI-BS-resistant sexual progeny, 26 were MAT1-1 and 34 were MAT12 (Table 3). Of the parasexual isolates, 21 were MAT1-1 and 28 were MAT1-2 ${ }^{15}$. The chi-square value of 0.002 and $P$ value of $0.98-0.95$ indicated that the segregation of the mating type in the BI-BS-resistant parasexual recombinants fitted that in the BI-BS-resistant sexual progeny.

\section{Discussion}

We demonstrated that the segregation ratio of the genetic character on pathogenicity and the mating type of parasexual recombinants were consistent with those of sexual progeny in the rice blast fungus $M$. oryzae. Heterokaryosis and a parasexual cycle have been reported in other filamentous fungi ${ }^{3,5,6,8,13,14,17,18,22,25}$, and parasexual recombination has proven to be a valuable tool in genetic analyses of filamentous fungi, particularly imperfect fungi. For example, the placement of adenine, lysine and white conidium gene markers in the same linkage group in Aspergillus niger was revealed through the parasexual cycle $^{10}$. The steps in the parasexual cycle are anastomosis, heterokaryosis, diploidization, and subsequently hap- loidization. New combinations of genes arise through haploidization, when the genes are in different linkage groups or mitotic crossing-over occurs ${ }^{10}$. Therefore, parasexual recombination may give rise to the segregation of genotypes in M. oryzae in a recombination manner similar to sexual events ${ }^{15}$. The segregation of avirulence to virulence in the progeny derived from crossing Y90-71 and 3514-R-2 on Hattan 3 was in a ratio of 95:90, and consistent with a $1: 1$ ratio $^{24}$, but that of the parasexual recombinants (BI-BS-resistant parasexual recombinants) on Hattan 3 was not consistent with a 1:1 ratio. These results suggested that Avr-Hattan 3 was in a linkage group with introduced antibiotic-resistance genes. While the segregation of avr/vir in the progeny derived from crossing Y90-71 and 3514-R-2, in BI-BS-resistant parasexual recombinants and in the BI-BS-resistant sexual progeny was consistent with a 1:1 ratio on line K59-1, which suggested that Avr-Pit was not in a linkage group with introduced antibiotic-resistance genes. Parasexuality in imperfect fungi is important in maintaining genetic diversity. It appears to be an adaptation that occurs predominantly in asexual fungi, such as the rice blast fungus. In plant pathogens, a parasexual cycle might lead to variable pathogenicity, which is a critical problem in controlling plant disease.

Although many comprehensive studies on parasexuality in the rice blast fungus have been conducted $^{3,5,6,8,13,14,20,22,25}$, little direct evidence exists of the parasexual cycle in nature. Nevertheless, parasexuality must be able to make a considerable contribution to genetic diversity in field populations of the rice blast fungus based on some observations ${ }^{2,25}$. Using DNA fingerprints, field populations of the rice blast fungus were not strictly clonal, and studies suggested parasexual and sexual recombination ${ }^{2,25}$. Further studies on the population structure and genetics of M. oryzae are needed to elucidate the role of parasexuality in nature.

\section{References}

1. Avalos, J., Geever, R. F. \& Case, M. E. (1989) Bialaphos resistance as a dominant selectable marker in Neurospora 
crassa. Curr. Genet., 16, 369-372.

2. Chen, Q. H., Wang, Y. C. \& Zheng, X. B. (2006) Genetic diversity of Magnaporthe grisea in China as revealed by DNA fingerprints haplotypes and pathotypes. $J$. Phytopathol., 154, 361-369.

3. Correll, J. C. et al. (2000) Characterization of Pyricularia grisea in the United States using independent genetic and molecular markers. Phytopathology, 90, 1396-1404.

4. Couch, B. C. \& Kohn, L. M. (2002) A multilocus gene genealogy concordant with host preference indicates segregation of a new species, Magnaporthe oryzae, from $M$. grisea. Mycologia, 94, 683-693.

5. Crawford, M. S. et al. (1986) Characterization of the heterokaryotic and vegetative diploid phases of Magnaporthe grisea. Genetics, 114, 1111-1129.

6. Fatemi, J. \& Nelson, R. R. (1978) Inter-isolate heterokaryosis in Pyricularia oryzae. Phytopathology, 68, 1791-1794.

7. Flor, H. H. (1956) The complementary genic systems in flax and flax rust. Adv. Genet., 8, 29-54.

8. Genovesi, A. D. \& Magill, C. W. (1976) Heterokaryosis and parasexuality in Pyricularia oryzae Cavara. Can. J. Microbiol., 22, 531-536.

9. Kimura, M. et al. (1995) A novel transformation system for Pyricularia oryzae: adhesion of regenerating fungal protoplasts to collagen-coated dishes. Biosci. Biotech. Biochem., 59, 1177-1180.

10. Lhoas, P. (1967) Genetic analysis by means of the parasexual cycle in Aspergillus niger. Genet. Res., 10, $45-61$.

11. Luo, C. X. et al. (2004) Identification of Magnaporthe oryzae avirulence genes to three rice blast resistance genes. Plant Dis., 88, 265-270.

12. Mandel, M. A. et al. (1997) Physical mapping of the Magnaporthe grisea AVR1-MARA locus reveals the virulent allele contains two deletions. Mol. Plant-Microbe Interact., 10, 1102-1105.

13. Namai, T. \& Yamanaka, S. (1982) Studies on variation in virulence of rice blast fungus, Pyricularia oryzae Cavara. I. Variant formation by the pairing-cultivation and -inoculation of two different pathogenic isolates. Ann. Phytopath. Soc. Jpn., 48, 466-470 [In Japanese with English summary].

14. Namai, T., Ehara, Y. \& Togashi, J. (1990) Changes in aggressiveness of a Pyricularia oryzae isolate (race 337) by successive passage on rice cultivars with different true resistance gene. Ann. Phytopath. Soc. Jpn., 56, 1-9 [In Japanese with English summary].

15. Noguchi, M. T., Yasuda, N. \& Fujita, Y. (2006) Evidence of genetic exchange by parasexual recombination and genetic analysis of pathogenicity and mating type of parasexual recombinants in rice blast fungus, Magnaporthe oryzae. Phytopathology, 96, 746-750.

16. Pall, M. L. \& Brunelli, J. P. (1993). A series of six compact fungal transformation vectors containing polylinkers with multiple unique restriction sites. Fungal Genet. Newsl., 40, 59-62.

17. Papa, K. E. (1978) The parasexual cycle in Aspergillus parasiticus. Mycologia, 70, 766-773.

18. Swart, K. et al. (2001) Genetic analysis in the asexual fungus Aspergillus niger. Acta Biol. Hung., 52, 335-343.

19. Valent, B., Farrall, L. \& Chumley, F. G. (1991) Magnaporthe grisea genes for pathogenicity and virulence identified through a series of backcrosses. Genetics, 127, 87-101.

20. Xia, J. Q. \& Correll, J. C. (1995) Examination of mitotic stability and hybridization potential between two genetically distinct haplotypes of Magnaporthe grisea. Exp. Mycol., 19, 171-177.

21. Yaegashi, H. (1981) Studies on the perfect stage of Pyricularia species. Bull. Tohoku Natl. Agric. Exp. Stn. Jpn., 63, 49-125.

22. Yamasaki, Y. \& Niizeki, H. (1965) Studies on variation of the rice blast fungus, Pyricularia oryzae Cav. I. Karyological and genetical studies on variation. Bull. Natl. Inst. Agric. Sci. Jpn. Ser. D, 13, 231-273.

23. Yasuda, N., Fujita, Y. \& Noguchi, M. (2004) Identification of avirulence genes in the rice blast fungus corresponding to three resistance genes in Japanese differentials. J. Gen. Plant Pathol., 70, 202-206.

24. Yasuda, N., Noguchi, M. T. \& Fujita, Y. (2005) Identification of an avirulence gene in the fungus Magnaporthe grisea corresponding to a resistance gene at the Pik locus. Phytopathology, 95, 768-772.

25. Zeigler, R. S. et al. (1997) Evidence of parasexual exchange of DNA in the rice blast fungus challenges its exclusive clonality. Phytopathology, 87, 284-294. 